Classification

Physics Abstracts

74.30E - 74.40

\title{
Specific heat measurements of organic superconductivity in (TMTSF) ${ }_{2} \mathrm{ClO}_{4}$
}

\author{
P. Garoche, R. Brusetti, D. Jérome \\ Laboratoire de Physique des Solides, Université Paris-Sud, 91405 Orsay, France \\ and K. Bechgaard \\ H. C. Oersted Institute, Universitetsparken 5, DK-2100 Copenhagen, Denmark
}

(Reçu le 30 novembre 1981, accepté le 12 janvier 1982)

\begin{abstract}
Résumé. - Nous avons effectué des mesures de la chaleur spécifique du supraconducteur organique $(\mathrm{TMTSF})_{2} \mathrm{ClO}_{4}$ entre 0,4 et $2 \mathrm{~K}$. La forte anomalie de la contribution électronique à la chaleur spécifique observée à $1,22 \mathrm{~K}$ a été attribuée à l'établissement de l'ordre supraconducteur tridimensionnel. En outre la très forte dépendance de la contribution électronique en champ magnétique au-dessus de 1,22 K n'est pas caractéristique d'un conducteur usuel dans un état normal. Un champ magnétique de $63 \mathrm{kOe}$ appliqué suivant l'axe $c^{*}$ des monocristaux stabilise un état fondamental du système présentant une très faible valeur de la densité d'états au niveau de Fermi (un état soit semiconducteur à petit "gap " soit semimétallique). On observe sous champ une transition isolantconducteur vers $1,4 \mathrm{~K}$. Nos résultats expérimentaux sont bien en accord avec l'existence d'un large domaine fluctuatif supraconducteur unidimensionnel.
\end{abstract}

\begin{abstract}
The specific heat of the organic superconductor (TMTSF) ${ }_{2} \mathrm{ClO}_{4}$ has been measured between 0.4 and $2 \mathrm{~K}$. A large anomaly of the electronic contribution at $1.22 \mathrm{~K}$ has been attributed to the onset of three-dimensional superconducting order. There is a large magnetic field dependence of the electronic contribution above $1.22 \mathrm{~K}$ which is not characteristic of a normal conducting state. The application of a magnetic field of $63 \mathrm{kOe}$ along the $c^{*}$-axis of the single crystals restores a ground state exhibiting a very low density of states at the Fermi level (a narrow gap semiconducting or a semimetallic state). A phase transition towards a conducting state with a finite density of states at Fermi level is visible around $1.4 \mathrm{~K}$. Our specific heat results support the existence of a wide 1-D superconducting fluctuating regime.
\end{abstract}

The physical properties of Quasi-One-Dimensional conductors are governed by several important concepts :

(i) As is well known [1] a strictly 1-D conductor with short-range interactions does not develop long-range order at any finite temperature.

(ii) However, if the thermal energy $k T$ is less than the energy characteristic of the intrachain interaction $k T_{1}$ significant short-range correlations can develop along the chain [2].

(iii) In a real system with a weak interchain coupling a phase transition occurs into a longrange 3-D ordered state at a finite temperature termed here $T_{3}$ which owing to the strong fluctua- 
tions may be considerably lower than the temperature $T_{1}$ below which 1-D short-range order is established. The temperature $T_{1}$ may be comparable with the 1-D mean field transition temperature where the 1-D mean field theory (neglecting thermal fluctuations of the order parameter) predicts the onset of long-range order [3, 4].

(iv) The theory of Quasi-One-Dimensional conductors predicts the existence of phase transitions driven by the minimization of the electron kinetic energy with the corresponding opening of an energy gap in the density of states at the Fermi level. The nature of the low temperature state may be either dielectric (a non-magnetic Peierls state or a spin density wave (SDW) Slater state) or superconducting (with either singlet or triplet spin pairing) [5].

The recent discovery of superconductivity in an organic conductor, the conducting chargetransfer salt (TMTSF) ${ }_{2} \mathrm{PF}_{6}$ [6], showing low dimensional features in its electronic properties has provided a unique opportunity to investigate the validity of the features of $1-\mathrm{D}$ theory mentioned above. Presently, the interpretation of the experimental results related to the establishment of superconductivity in (TMTSF) ${ }_{2} \mathrm{PF}_{6}$ is somewhat controversial. On the one hand, the Orsay group has claimed [7] that strong superconducting 1-D precursor effects exist at temperatures significantly larger than the onset of 3-D order. Fluctuations effects have been claimed up to $30 \mathrm{~K}$ or so whereas $3-\mathrm{D}$ order occurs only below $T_{3} \approx 1.2 \mathrm{~K}$. On the other hand the UCLA-IBM team has proposed an alternative explanation to the very peculiar low temperature properties of (TMTSF) ${ }_{2} \mathrm{PF}_{6}$ and related compounds in terms of very high mobility carriers in a 2-D conductor [8].

Since the Physics of Q-1-D conductors implies the existence of two energy scales, one which is related to the large intrachain coupling and the other which corresponds to the much weaker interchain interaction we deemed it necessary to clarify this point on an experimental basis through an investigation of the thermodynamic properties of the 3-D superconducting transition.

It is the purpose of this article to report the first observation of Organic Superconductivity in (TMTSF $)_{2} \mathrm{ClO}_{4}$ via specific heat measurements. The specific heat of (TMTSF) ${ }_{2} \mathrm{ClO}_{4}$ single crystals has been studied between 0.4 and $2 \mathrm{~K}$ with low frequency calorimetric technique described elsewhere [9].

Eight single crystals of total weight $3.2 \mathrm{mg}$ have been aligned and glued on a thin sapphire slab placed in thermal contact with a heat sink. Measurements of the specific heat have been performed in zero magnetic field $(H \lesssim 0.2 \mathrm{Oe})$ and in fields of up to $63 \mathrm{kOe}$ approximately aligned along the transverse $c^{*}$-axis of the samples $\left( \pm 5^{\circ}\right)$. The data of molar specific heat after subtraction of the sample holder contribution determined in a separate run, are displayed in figure 1 . On a $C / T$ versus $T^{2}$ plot (where a mole of (TMTSF) ${ }_{2} \mathrm{ClO}_{4}$ contains $N$ (Avogadro number) molecular units (TMTSF) ${ }_{2} \mathrm{ClO}_{4}$, figure $1 a$ ), a very large specific heat anomaly is visible around $T \approx 1.2 \mathrm{~K}$ i.e. the critical temperature for superconductivity. Above $T^{2}=1.5 \mathrm{~K}^{2}$, the specific heat obeys the classical relation $C / T=\gamma+\beta T^{2}$ where $\gamma$ is proportional to the electronic density of states at the Fermi level (the electronic specific heat) and $\beta$ is inversely proportional to the (Debye temperature) $^{3}$. From figure $1 a$ we derive a lattice contribution $\beta=11.4 \mathrm{~mJ} / \mathrm{mole} . \mathrm{K}^{4}$. Following the definition of the lattice specific heat $C_{\mathrm{lat}}=12 \pi^{4} \frac{N k_{\mathrm{B}}}{5}\left(\frac{T}{\theta_{\mathrm{D}}}\right)^{3}$ we derive a Debye temperature $\theta_{\mathrm{D}}=213 \mathrm{~K}$ from the data shown on figure $1 a$. In this derivation of $\theta_{\mathrm{D}}$ we have used the fact that a unit cell contains 57 atoms. Figure $1 b$ displays the molar electronic contribution to the specific heat after removal of the lattice contribution previously derived. The $C / T$ versus $T$ plot of figure $1 b$ allows a very accurate determination of both the critical temperature and the specific heat jump at the transition. Approximating the specific heat anomaly in the transition region by the usual triangular approximation with an equal area argument [10], we derive from the data of figure $1 b, T_{\mathrm{c}}=1.22 \mathrm{~K}, \gamma=10.5 \mathrm{~mJ} / \mathrm{mole} . \mathrm{K}^{2}, \Delta C=21.4 \mathrm{~mJ} / \mathrm{mole} . \mathrm{K}$ and $\Delta C / \gamma T_{\mathrm{c}}=1.67$ a ratio which is slightly larger than the prediction of the BCS theory, namely 

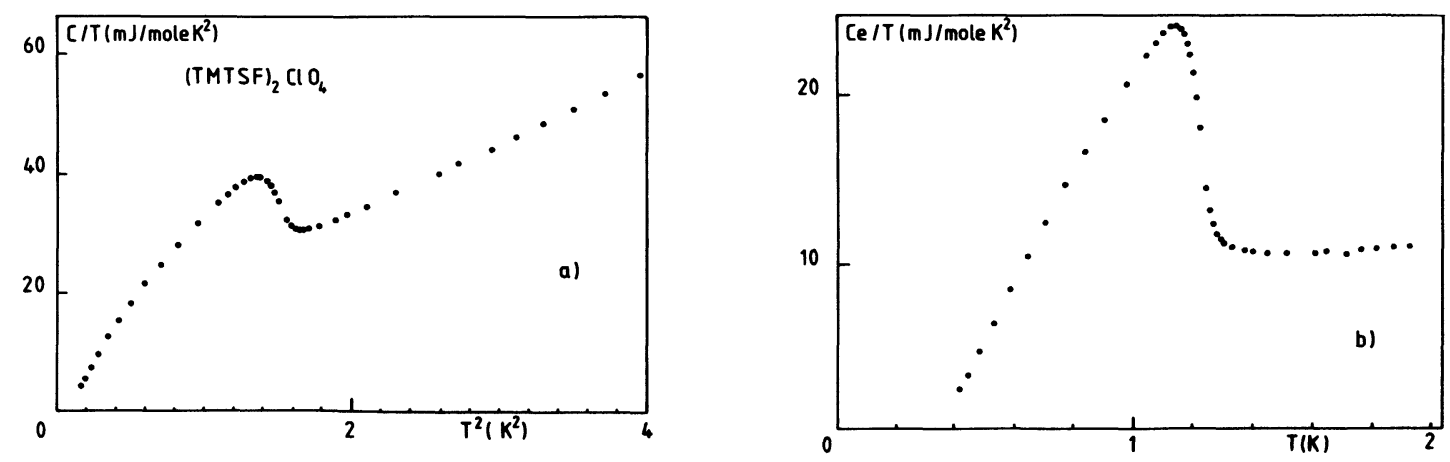

Fig. 1. - Temperature dependence of the molar specific heat of (TMTSF) ${ }_{2} \mathrm{ClO}_{4}$ in zero magnetic field, lattice and electronic contribution $(a)$ and electronic contribution after subtraction of the lattice contribution $(b)$.

1.43 [11]. The value of $T_{\mathrm{c}}$ obtained in the present experiment is in fair agreement with resistivity [12, 13] and Meissner effect data [14, 15].

The bulk and homogeneous nature of the superconducting state below $T_{\mathrm{c}}$ are supported by the sharpness $\left(\frac{\Delta T_{\mathrm{c}}}{T_{\mathrm{c}}} \sim 4 \%\right)$ and the amplitude of the specific heat anomaly presented in figure $1 b$.

Furthermore, the data of figure $1 b$ enable a thermodynamic characterization of the superconducting state below $T_{\mathrm{c}}$. At $T=0$, the free energy difference between the normal and superconducting states gives the condensation energy $H_{0}^{2} / 8 \pi$. Therefore from

$$
H_{0}^{2} / 8 \pi=\int_{0}^{T_{\mathrm{c}}}\left(C_{\mathrm{es}}-C_{\mathrm{n}}\right) \mathrm{d} T,
$$

we obtain $H_{0}=44 \pm 2$ Oe. Moreover, a fit of the data in figure $1 b$ with an exponential temperature dependence of the specific heat at low temperatures leads to an energy gap $2 \delta \approx 4 \mathrm{~K}$ which is in accord with the low value of the critical temperature. However, the quasi-BCS value for the ratio of the gap to the critical temperature may be forfuitous as the superconducting transition observed at $1.22 \mathrm{~K}$ is within the spirit of 1-D Physics related to the long-range interchain ordering of the phase of the superconducting order parameter. Figure $1 b$ allows a direct determination of the density of states at the Fermi level. According to the relation

$$
N\left(E_{\mathrm{F}}\right)=3 \gamma / 2 \pi^{2} k_{\mathrm{B}}^{2},
$$

$N\left(E_{\mathrm{F}}\right)=2.1$ states/eV . molecule is derived from the data of figure $1 b$.

We may notice that the experimental value of $N\left(E_{\mathrm{F}}\right)$ is quite large. It is about the density of states which is observed in transition metal alloys and intermetallic A 15 structures [16]. In addition, even if the interpretation of the specific heat anomaly is performed in terms of McMillan's theory of strong coupling superconductors [16] the bare density of states obtained after taking into account the enhancement factor $1+\lambda=1.44$ is still 1.5 states $/ \mathrm{eV}$. molecule (with $T_{\mathrm{c}}=1.22 \mathrm{~K}$, $\theta_{\mathrm{D}}=213 \mathrm{~K}$ and $\mu^{*}=0.1$ in McMillan's formula for $\left.T_{\mathrm{c}}[16]\right)$. For the sake of completeness note that the density of states inferred from a tight binding model calculation is

$$
N\left(E_{\mathrm{F}}\right) \approx 2 \text { states/eV. molecule, }
$$

assuming a quarter-filled band of width $0.8 \mathrm{eV}$. It is however by no means obvious that such a simple model can provide a relevant picture. 
Under transverse magnetic fields $\left(H / / c^{*}\right)$, the transition temperature is depressed at a rate of $\mathrm{d} H_{\mathrm{c} 2}^{\perp} / \mathrm{d} T=880 \pm 20 \mathrm{Oe} / \mathrm{K}$. When the magnetic field exceeds one kOe or so, the specific heat anomaly is no longer observable in the $0.4-2 \mathrm{~K}$ temperature range. In the low field regime, i.e. $1 \mathrm{kOe}$ or so, the electronic specific heat follows the law $C / T=\gamma+\beta T^{2}$ with the parameters $\gamma$ and $\beta$ approximately equal to the values deduced at $T^{2}>2 \mathrm{~K}^{2}$ in zero field, from figure $1 a$.

However drastic changes in the temperature dependence of the electronic specific heat are observed in higher fields. Figure 2 shows the behaviour of the specific heat for a field of $63 \mathrm{kOe}$. In figure $2 a$, the total sample specific heat (including the lattice contribution) is displayed on a $C / T$ versus $T^{2}$ plot. After subtraction of a lattice contribution derived from the zero field data we get the electronic contribution plotted in figure $2 b$. This figure exhibits several striking features :

(i) The electronic specific heat vanishes as $T$ goes to zero implying therefore that the ground state of (TMTSF) ${ }_{2} \mathrm{ClO}_{4}$ under high magnetic field contains only a very low density of states at the Fermi level. Obviously this low density of states cannot be attributed to a single particle gap resulting from superconductivity since $H_{\mathrm{c} 2}^{\perp}\left(H / / c^{*}\right)$ is about $0.5 \mathrm{kOe}$ for (TMTSF) ${ }_{2} \mathrm{ClO}_{4}$ around $0.4 \mathrm{~K}$.

Therefore, we are forced to conclude that the data of figure $2 b$ imply the stabilization of a low density of states phase (a semiconducting or more likely a semimetallic phase) in (TMTSF) ${ }_{2} \mathrm{ClO}_{4}$ at very low temperatures under high field.

(ii) Going up in the temperature, figure $2 b$ reveals a specific heat anomaly at $T \approx 1.4 \mathrm{~K}$ which marks the existence of the phase transition between the low temperature low density of carriers state and the high temperature conducting state.

(iii) The electronic specific heat of the conducting state above 1.4 is about $70 \%$ larger than the corresponding value in zero field. Consequently the density of states of (TMTSF) ${ }_{2} \mathrm{ClO}_{4}$ measured at $T \approx 2 \mathrm{~K}$ is enhanced by the application of a magnetic field. This point will be made clearer in a forthcoming study of the specific heat at higher temperatures [17].
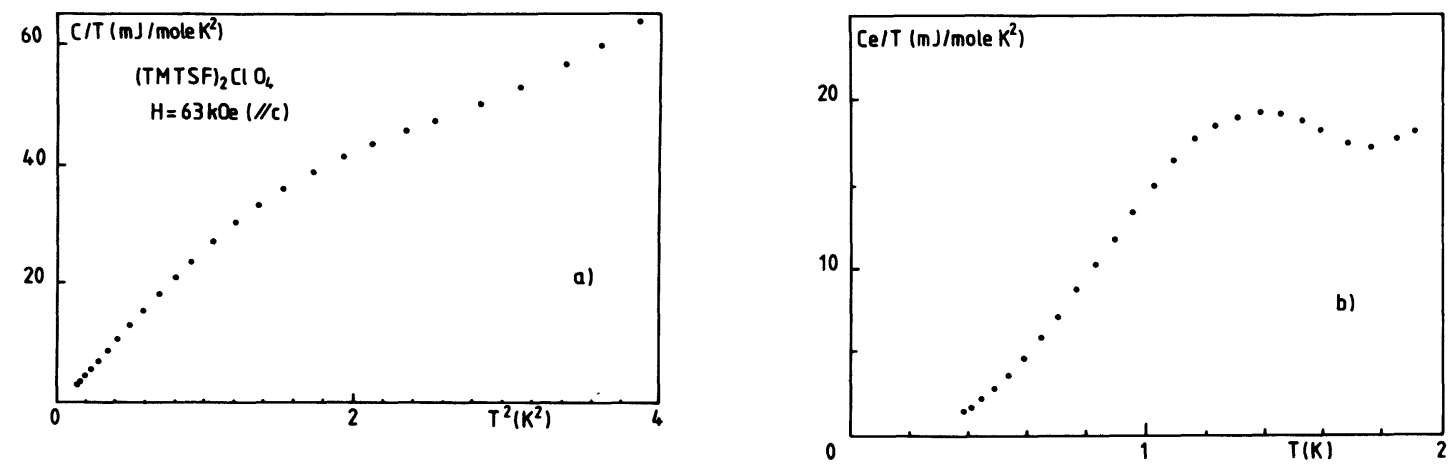

Fig. 2. - Temperature dependence of the molar specific heat of (TMTSF) ${ }_{2} \mathrm{ClO}_{4}$ under a magnetic field of $63 \mathrm{kOe}$ applied along the $c$-direction, lattice plus electronic contribution (a), electronic contribution (b).

We shall now proceed to a discussion of the high field specific heat data of (TMTSF) ${ }_{2} \mathrm{ClO}_{4}$ within the framework of the electronic properties of the other members of the (TMTSF) ${ }_{2} \mathrm{X}$ series. We first note that the low temperature behaviour of (TMTSF) ${ }_{2} \mathrm{ClO}_{4}$ at the ambient pressure closely resembles that of the pressure stabilized conducting state of (TMTSF) ${ }_{2} \mathrm{PF}_{6}($ at $P>9 \mathrm{kbar}$ or so). Consequently, we believe that the phase transition observed by NMR in (TMTSF) ${ }_{2} \mathrm{PF}_{6}$ under pressure and high magnetic field at about $2 \mathrm{~K}$ [18] bears striking similarities with the transition observed in the present study at $1.4 \mathrm{~K}$ and $63 \mathrm{kOe}$ for (TMTSF) ${ }_{2} \mathrm{ClO}_{4}$. 
Secondly the present study clarifies the unusual behaviour of the de Haas-Shubnikov effect observed at $1.1 \mathrm{~K}$ in (TMTSF) ${ }_{2} \mathrm{PF}_{6}$ under pressure [19], namely that quantum oscillations at $T=1.1 \mathrm{~K}$ are observed only when a low $N\left(E_{\mathrm{F}}\right)$ (semimetallic) state is stabilized by the application of a large magnetic field corresponding to an onset field along the $c^{*}$-axis of about $60 \mathrm{kOe}$.

The restoration of a semimetallic state under high magnetic field provides a very simple interpretation of the large transverse magnetoresistance for $H / / c^{*}$ which is observed in the helium temperature range in (TMTSF) ${ }_{2} \mathrm{ClO}_{4}$ or in the pressure stabilized conducting phase of (TMTSF) ${ }_{2} \mathrm{PF}_{6}$ [20] or (TMTSF) ${ }_{2} \mathrm{AsF}_{6}$ [21]. For fields aligned with the $b$-direction, the magnetoresistance is significantly smaller [21] in agreement with an interchain coupling much weaker in the $c$-direction than along the $b$-direction [22]. The restoration of a semimetallic state (if it is at all possible) would therefore require a field much larger than say $100 \mathrm{kOe}$. Our experimental results suggest that the restoration of a semimetallic state is a direct consequence of the suppression of the 1-D superconducting fluctuating state.

The semimetallic state may be characterized by Spin Density Waves similar to the ambient pressure semiconducting state of (TMTSF) ${ }_{2} \mathrm{PF}_{6}$. NMR experiments nearing completion should be able to settle this question soon [23].

Another highlight of the specific heat behaviour is the increase of $N\left(E_{\mathrm{F}}\right)$ at $T \approx 2 \mathrm{~K}$ under magnetic field. We believe this can be understood as the suppression under high fields of the pseudo-gap of 1-D superconducting fluctuating origin. The increase of density of states under magnetic field is actually in qualitative agreement with the effect of a magnetic field on the tunnelling characteristics in (TMTSF) ${ }_{2} \mathrm{PF}_{6}$ at $12 \mathrm{kbar}$ [24].

Finally, the 3-D ordering of the superconducting order parameter at $1.2 \mathrm{~K}$ in (TMTSF) ${ }_{2} \mathrm{ClO}_{4}$, which requires a small condensation energy $H_{0}^{2} / 8 \pi$ of $\approx 2 \times 10^{16} \mathrm{eV} / \mathrm{mole}$ appears to be well approximated by a nearly mean field treatment. This is however expected if the critical region in temperature is small enough. Indeed, the analysis of the paraconductivity in (TMTSF) ${ }_{2} \mathrm{PF}_{6}$ [7] above $T_{3}$ has located the cross-over temperature between the 1-D regime and a higher dimensionality regime at $T<T_{3}$ as being at around $1.7 \mathrm{~K}$. Consequently there are no reasons to observe prominent fluctuation effects in the immediate vicinity of the transition at $T_{3}$ and corresponding large deviations from a mean field behaviour.

In conclusion, the specific heat investigation of superconductivity in (TMTSF) ${ }_{2} \mathrm{ClO}_{4}$ supports the applicability of the concepts of One-Dimensional Physics to this series of 1-D conductors and reinforces the point of view incorporating 1-D superconducting fluctuations in a large domain of temperature where they are predominant. Furthermore this investigation has proved the establishment of a semiconducting (or semimetallic) ground state under high magnetic field.

Acknowledgments. - We thank H. J. Schulz and C. Bourbonnais for fruitful discussions. We also acknowledge stimulating and fruitful discussions with M. Ribault.

\section{References}

[1] Landau, L. D. and Lifshitz, E. M., Statistical Physics (Pergamon, New York) 1958, p. 482.

[2] Scalapino, D. J., Sears, M. and Ferrell, R. A., Phys. Rev. B 6 (1972) 3409.

[3] Scalapino, D. J., ImRY, Y. and Pincus, P., Phys. Rev. B 5 (1975) 2042.

[4] Schulz, H. J., Proceedings of the International Conf. on Low Dimensional Conductors, Boulder (1981), to be published in Mol. Cryst. Liq. Cryst.

[5] SOlyom, J., Adv. Phys. 28 (1979) 201.

[6] Jérome, D., Mazaud, A., Ribault, M., Bechgaard, K., J. Physique-Lett. 41 (1980) L-95.

[7] Schulz, H. J., Jérome, D., Mazaud, A., Ribault, M. and BechgaArd, K., J. Physique 42 (1981) 991.

[8] Greene, R. L., Haen, P., Huang, S. Z., Engler, E. M., Choi, M. Y. and Chaikin, P. M., Proceedings of the Boulder Conference 1981, to be published in Mol. Cryst. Liq. Cryst. 
[9] Manuel, P. and Veyssié, J. J., Phys. Rev. B 14 (1978) 78 and

Garoche, P., Thesis, University of Paris-Sud (1981), unpublished.

[10] Garoche, P., Manuel, P., Veyssié, J. J. and Molinié, P., J. Low Temp. Phys. 30 (1978) 325.

[11] TinkhaM, M., Introduction to Superconductivity (Mc-Graw Hill, New York) 1975.

[12] Bechgaard, K., Carneiro, K., Olsen, M., Rasmussen, F. B. and Jacobsen, C. S., Phys. Rev. Lett. 46 (1981) 852.

[13] Parkin, S. S. P., Ribault, M., Jérome, D. and Bechgaard, K., J. Phys. C, Solid State 14 (1981) L-445 and J. Phys. C, Solid State (1981), to be published.

[14] Schwenk, H., Neumaier, K., Andres, K. and Wudl, F., Proceedings of the Boulder Conference 1981, to be published in Mol. Cryst. Liq. Cryst.

[15] Ribault, M. and Mailly, D., to be published (1982).

[16] McMillan, W. L., Phys. Rev. 167 (1968) 331.

[17] Brusetti, R. and Garoche, P., to be published.

[18] Azevedo, L. J., Schirber, J. E., Greene, R. L. and Engler, E. M., Physica 108B (1981) 1183.

[19] Kwak, J. F., Schirber, J. E., Greene, R. L. and Engler, E. M., Phys. Rev. Lett. 46 (1981) 1296.

[20] Jérome, D., Chem. Scr. 17 (1981) 13.

[21] Brusetti, R., Ribault, M., Jérome, D. and Bechgaard, K., J. Physique, to be published (1982).

[22] Thorup, N., Rindorf, G., Soling, H. and BechgaArd, K., Acta Crystallogr. B 37 (1981) 1236 and WUDL, F., preprint (1981).

[23] Takahashi, T., Jérome, D. and BechgaARd, K., to be published (1982).

[24] More, C., Roger, G., Sorbier, J. P., Jérome, D., Ribault, M. and BechgaArd, K., J. Physique-Lett. 42 (1981) L-313. 